\title{
Schwangerschaft möglichst in Remission planen
}

\begin{abstract}
Eine Schwangerschaft bei Frauen mit chronisch entzündlicher Darmerkrankung unterscheidet sich bei geringer Krankheitsaktivität oder in Remission nicht von dem bei gesunden Frauen. Die übliche medikamentöse Basistherapie kann ohne erhöhtes Risiko für Mutter und Kind fortgesetzt werden.
\end{abstract}

Im akuten Schub einer CED kann bei den betroffenen Frauen die Fertilität herabgesetzt sein. Die Fruchtbarkeit in der Remission unterscheide sich dagegen nicht von Gesunden, erklärte Prof. Dr. Axel Dignaß, Frankfurt am Main. Weil im akuten Entzündungsschub außerdem die Wahrscheinlichkeit für Fehl- und Frühgeburt sowie weitere Komplikationen steigt, sollten CED-Patienten Schwangerschaften möglichst in Remissionsphasen planen.
Bei der Behandlung schwangerer Frauen mit CED stellen etablierte Basismedikamente wie Sulfasalazin, 5-ASA, Budesonid (z.B. Budenofalk ${ }^{\oplus}$ ) oder andere Steroide kein Risiko für das Kind dar. Es gebe weder vermehrt Fehlgeburten noch Fehlbildungen, so Dignaß. Allerdings müsse gehäuft mit Frühgeburten und einem verminderten Geburtsgewicht der Kinder gerechnet werden. Dies sei weniger der Medikation als der chronischen Krankheit geschuldet.

\section{Basismedikation fortsetzen}

Weil ein akuter Schub während der Schwangerschaft ein erhebliches Risiko darstellen kann, sollte die Behandlung mit der Basismedikation fortgesetzt werden, wenn dies zum Remissionserhalt notwendig ist. Kommt es während der
Schwangerschaft zu einem Krankheitsschub, erfolgt die Behandlung mit 5-ASAPräparaten und Kortikosteroiden in üblicher Weise. Laut ECCO gelten auch die Immunsuppressiva Azathioprin und 6-Mercaptopurin als sicher, wenn eine klare Indikation zur Immunsuppression besteht. Wenn möglich, sollte im dritten Trimenon keine anti-TNF-Therapie erfolgen, da diese Substanzen plazentagängig sind und das Immunsystem des Kindes beeinflussen könnten. Methotrexat ist kontraindiziert, eine Behandlung mit Methotrexat sollte bereits drei bis zwölf Monate vor geplanter Schwangerschaft beendet werden.

Falk Kolloquium "Herausforderungen und Lösungen für Gastroenterologen und Chirurgen in der Viszeralmedizin", 26.-27. Juni 2015, Berlin

\section{Morbus Crohn: Analfisteln sphinkterschonend beseitigen}

\section{Treten bei einem Morbus Crohn} anale Fisteln auf, sollte deren operativer Fistelverschluss angestrebt werden. Voraussetzung für den Erfolg ist die optimale Therapie der Grunderkrankung.

„Es kommen nur sphinkterschonende Operationen in Betracht", betonte Dr. Roland Scherer, Berlin. Zunächst besteht die Möglichkeit, die Fistel mit einem Faden zu drainieren und die konservative Therapie zu optimieren, bis die Entzün- dung zur Ruhe gekommen ist. Die dauerhafte Fadendrainage wird wenig toleriert, besonders wenn mehrere Fisteln vorliegen.

Scherer stellte zwei Methoden für den Fistelverschluss vor. Der Analfistel-Plug ist ein fadenförmiger Pfropfen aus Schweinekollagen, der keine Fremdkörper- oder Entzündungsreaktion nach Implantation auslöst. Das Material wird durch die Fistel gezogen und an den Fistelausgängen gekürzt. In Studien werden Verschlussraten von etwa $40-50 \%$ berichtet - allerdings ist die Datenlage für Mor-

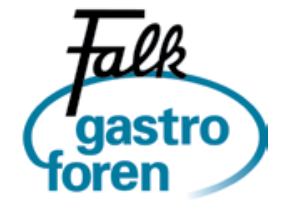

Besuchen Sie das nächste Falk Gastro Forum "Neue und bewährte Konzepte für den Darm" am Samstag, den 10. Oktober 2015 in Rostock; Infos: www.drfalkpharma.de/veranstaltungen bus Crohn unzureichend. Vorteile sind die einfache Operationstechnik ohne Risiko für die Kontinenz.

Eine chirurgisch anspruchsvolle Methode ist LIFT (Ligation of the Intersphincteric Fistula Tract). Dabei wird über einen Zugang zwischen innerem und äußerem Schließmuskel der Fistelgang aufgesucht, durchtrennt und mit chirurgischen Nähten verschlossen. Die Erfolgsraten werden über alle Patienten mit etwa 75\% angegeben, wobei für Crohn-Patienten keine ausreichenden Daten vorliegen. Bei schweren, therapierefraktären Verläufen könne die Proktektomie für die Patienten ,ein Segen sein", so Scherer.

Falk-Kolloquium "Herausforderungen und Lösungen für Gastroenterologen und Chirurgen in der Viszeralmedizin", 26.-27. Juni 2015, Berlin 